\title{
Rursus
}

Russus

Poiétique, réception et réécriture des textes antiques

$1 \mid 2006$

Le modèle animal (I)

\section{L'enseignement des langues anciennes dans huit des pays entrés dans la communauté européenne en 2004}

Michèle Biraud et Dagmar Muchnova

\section{(2) OpenEdition}

Journals

Édition électronique

URL : http://journals.openedition.org/rursus/53

DOI : 10.4000/rursus.53

ISSN : 1951-669X

Éditeur

Université Nice-Sophia Antipolis

Référence électronique

Michèle Biraud et Dagmar Muchnova, "L'enseignement des langues anciennes dans huit des pays entrés dans la communauté européenne en 2004 », Rursus [En ligne], 1 | 2006, mis en ligne le 09 juillet 2006, consulté le 19 avril 2019. URL : http://journals.openedition.org/rursus/53 ; DOI : 10.4000/ rursus. 53

Ce document a été généré automatiquement le 19 avril 2019

Rursus 


\title{
L'enseignement des langues anciennes dans huit des pays entrés dans la communauté européenne en 2004
}

\author{
Michèle Biraud et Dagmar Muchnova
}

1 Voici les résultats d'une enquête que nous avons menée sur les pays entrés dans la communauté européenne en mai 2004. Notre but a été de chercher des renseignements sur la place du latin et du grec ancien dans l'enseignement du second degré et sur la formation des maîtres, sur l'organisation des études en langues anciennes à l'université et leur niveau, sur les effectifs d'étudiants et les débouchés.

2 Nous avons d'abord fait un inventaire des universités où l'on enseigne les langues anciennes dans ces dix pays, en notant sur le Web les adresses de leurs sites et les e-mails de professeurs avec qui prendre contact (voir liste en annexe [1]). Puis nous avons rédigé un questionnaire, le plus factuel possible, et une réponse au questionnaire pour la France, pour que l'information soit réciproque. Nous avons envoyé le tout, en français et en anglais, à tous ces contacts potentiels. Notre premier envoi, en janvier, n'ayant eu aucun succès, nous l'avons renouvelé, cette fois en indiquant le sujet de notre courriel en latin, et avons obtenu une douzaine de réponses et l'adresse de nouveaux contacts. Avec les réponses de ces derniers, nous disposons d'informations sur près de vingt universités, soit un peu plus de la moitié des universités où l'on étudie les langues anciennes dans ces dix pays.

3 Plusieurs de ces pays ont, depuis le XIX siècle ou le début du XX $\mathrm{XX}^{\mathrm{e}}$ siècle, une société savante dédiée à l'étude des langues classiques (qui est membre de la F.I.E.C.) et des associations de professeurs antiquisants (enseignement secondaire et supérieur associés) : à Tartu, en Estonie, the Karl Morgenstern Classical Society, à Budapest, la société hongroise d'études classiques et la société des professeurs sur l'Antiquité classique (Okortudomànyi Tàrsasàg), à Varsovie, la Société polonaise de Philologie Classique (Polskie towarzystwo Filologiczne), à Prague, l'Union des philologues classiques 
(Jednota Klasickych Filologu), fondée en 1872, et l'ALFA (Association des professeurs de langues classiques), à Ljubljana, en Slovénie, la Societas sloveniae studiis antiquitatis et humanitatis investigandis...

4 Certaines de ces universités sont très anciennes. Ainsi, à Vilnius, le département de philologie classique faisait partie dès l'origine de l'université, qui a été fondée en 1579. Plus ancienne encore, l'Université Charles de Prague, fondée en 1348. A Budapest, le Cardinal Pierre Pazmany a fondé l'université au XVII ${ }^{e}$ siècle, puis il y a eu une scission après la deuxième guerre mondiale, une université laïque, baptisée du nom d'un scientifique hongrois, Eötvos Lörand, ayant repris la plupart des enseignements, mais l'université catholique, qui a conservé le nom de son fondateur, poursuit ses enseignements dans de nombreux domaines autres que la théologie, dont la philologie classique. Pour l'historique, nous nous en tiendrons à ces exemples représentatifs. Il faut toutefois ajouter que, dans certains pays, le nombre d'universités enseignant les langues classiques a diminué après la seconde guerre mondiale. Par exemple, il existait, avant la guerre, en Lituanie, un enseignement des langues anciennes aussi à l'Université Vytautas le Grand de Kaunas, puis tout a été concentré à l'Université de Vilnius.

Huit des dix pays qui ont rejoint la Communauté Européenne en 2004 avaient passé plus de quarante ans dans le bloc soviétique (tous sauf Malte et Chypre). La période socialiste y avait sérieusement compromis l'enseignement des lettres classiques et sa réputation. Au niveau du lycée, le grec avait été éliminé totalement et le latin l'avait été plus ou moins (sauf une courte période de revitalisation du latin dans les années soixante en Tchécoslovaquie). Ceci a amené une limitation des études universitaires, puisque l'on $\mathrm{n}$ 'avait plus besoin que de très peu de professeurs de lycée : ainsi, en Tchécoslovaquie, on n'ouvrait donc le latin qu'une fois tous les deux ou trois ans et le grec encore beaucoup plus rarement. Dans les autres pays, la situation a été plus ou moins comparable.

6 L'insertion des langues anciennes se fait selon les universités dans des Instituts ou des Facultés différents, ce qui est soit la trace de cette histoire mouvementée du dernier demi-siècle (où elles étaient considérées au mieux comme disciplines auxiliaires de l'histoire, de la philosophie ou de la littérature), soit la marque de la faiblesse de leurs effectifs, qui oblige à des apparentements divers. Nous en donnerons seulement quatre ou cinq exemples : à Cracovie, « l'institut de philologie classique » fait partie de la "Faculté de philologie étrangère ", à Gdansk, de la « Faculté de philologie et d'histoire ", à Ceske Budejovice (République tchèque), d'un département «d'histoire ancienne et philologie classique ", à Bratislava, d'un département de "philologie classique et sémitique ", à Chypre, d'un « département d'études classiques et philosophie ».

7 Nous allons commencer par un descriptif pays par pays, puis nous évoquerons les contenus d'enseignement et les débouchés, et nous soulignerons les corrélations de facteurs qui nous paraissent les plus significatives.

Il nous semble qu'il y a au moins un double enseignement à tirer de cette enquête: essayer d'éviter certains écueils dans le devenir de nos études en France (comme le serait l'affaiblissement de l'enseignement du latin au collège ou du grec au lycée, ou la renonciation à l'association du français aux langues anciennes dans les concours de recrutement); envisager des échanges intra-communautaires d'informations et des projets communs pour la promotion de l'enseignement des langues anciennes au niveau européen. 


\section{La situation dans chacun des huit pays}

Dix pays sont entrés dans la communauté européenne en 2004, mais les universitaires de Lituanie et de Malte n'ont pas répondu à notre questionnaire. Le descriptif de chaque pays présente une rapide description de l'état de l'enseignement dans le second degré, quelques indications sur la formation des professeurs, une analyse plus détaillée des cursus de spécialité langues anciennes, et une présentation de l'enseignement pour les non-spécialistes. Ces données sont récapitulées et complétées par des tableaux en annexe [2].

\section{En république tchèque et en république slovaque}

10 Nous exposons en même temps la situation en Tchéquie et en Slovaquie puisque 1 'organisation contemporaine des études classiques à l'Université est issue d'une longue association de ces deux pays, d'abord sous l'Empire Autrichien, puis dans l'Empire Austro-Hongrois; en 1918 fut constituée la République Tchécoslovaque qui, désunie temporairement sous l'occupation nazie, fut définitivement divisée en Tchéquie et Slovaquie en 1993.

11 Jusqu'en 1989, il n'existait que trois universités où l'on enseignait le latin : Prague, Brno et Bratislava. Depuis, quatre nouveaux Départements d'Études Classiques ont été créés, en Tchéquie, dans les universités de Ceske Budejovice et Olomouc, en Slovaquie, dans les universités de Trnava et de Presov. De nos jours, on enseigne donc en tant que matière principale le latin dans quatre universités en Tchéquie et trois en Slovaquie, et le grec dans deux universités en Tchéquie et une en Slovaquie.

\section{L’enseignement secondaire}

12 Les lycéens acquièrent des connaissances de littérature et de philosophie antiques $(20 \mathrm{H}$ au lycée) et d'histoire de l'antiquité ( $20 \mathrm{H}$ au collège, $30 \mathrm{H}$ au lycée). La langue grecque $\mathrm{n}$ 'est enseignée dans le secondaire ni en Slovaquie ni en Tchéquie (sauf une ou deux exceptions). En Tchéquie, la langue latine peut être étudiée soit - assez rarement - en matière obligatoire, soit - plus souvent - en option, pendant une à deux années à raison de $2 \mathrm{H}$ par semaine, donc en concurrence avec la conversation anglaise, l'étude approfondie de l'informatique, des travaux de laboratoire en biologie etc. (il est clair que la position du latin est peu favorable), soit comme matière facultative, donc «en plus " des heures obligatoires et optionnelles. En Slovaquie, certains lycées offrent une ou deux années de langue latine.

\section{La formation au métier d'enseignant et le recrutement des professeurs}

Pour devenir professeurs de lycée, les étudiants font, en même temps que leurs études de grec et/ou latin, un court cursus spécial comprenant de la psychologie, de la pédagogie, de la didactique et un stage en lycée. Le Département d'Etudes Classiques assure, dans ce minimum pédagogique, la didactique du latin (et du grec), les autres matières étant assurées par le Département de Pédagogie et de Psychologie de la Faculté des Lettres. 

En Tchéquie, les Facultés pédagogiques forment les enseignants de collèges et de lycées dans les matières dites scolaires (histoire, géographie, mathématiques, etc.; donc pas de grec, de copte, de sanscrit etc.). Le latin y est enseigné seulement comme matière non principale (à l'exception de la Faculté pédagogique de Ceske Budejovice), dans le cursus de l'histoire par exemple. Il n'existe pas de concours de recrutement des professeurs au niveau national.

\section{Le cursus universitaire de spécialité Etudes classiques}

En Tchéquie, les quatre universités ont un examen d'entrée (ou bien une interview sur la motivation des candidats, à Ceske Budejovice), alors qu'en Slovaquie, deux universités sur trois ont un examen d'entrée (sauf Trnava). L'examen d'entrée est soit écrit, soit oral, soit oral et écrit :

- a) en latin : traduction d'un texte latin inconnu (morphologie et un peu de syntaxe), littérature, culture, histoire de l'antiquité, grammaire tchèque, connaissances générales ;on peut être admis à la faculté sans avoir étudié le latin au lycée ; l'essentiel est d'avoir réussi aux examens d'entrée (on peut prendre des leçons privées de latin ou être autodidacte) ;

- b) en grec (si le grec est enseigné en tant que matière principale), le test est le même que pour le latin (mutatis mutandis), mais aucune connaissance de la langue grecque n'est exigée.

La Tchéquie et la Slovaquie (aussi bien que les autres pays) subissent de grands changements dus à l'adoption des conventions de Bologne, qui y seront généralement réalisées selon la formule « licence en trois ans + magister en deux ans + doctorat en trois ans ». Le progrès dans l'adoption du nouveau système est différent selon les universités. L 'ancien système qui est encore en vigueur dans certaines Universités consiste en un cursus de cinq ans d'études obligatoires qui se terminent par l'obtention du diplôme Magister, comprenant un premier cycle de deux ans et un second cycle de trois ans. Ceux qui veulent faire de la recherche ou enseigner à l'Université suivent un troisième cycle de trois ans (terminé par le diplôme Ph. D.)

études sont organisées d'habitude sur la base de deux matières principales. Le latin peut être choisi comme matière principale et associé à une autre matière enseignée à la Faculté des Lettres (par ex. latin - français, latin - tchèque, latin - histoire), qui est, à Prague et à Brno, au choix de l'étudiant; ailleurs, les associations de matières sont prescrites par le Ministère de l'Education (conformément à la suggestion de la faculté). Le cursus de latin comprend un cours obligatoire de langue grecque (120-240 H en tout), souvent des cours ou séminaires obligatoires de littérature grecque $(30 \mathrm{H}$ en tout) et $\mathrm{d}$ 'histoire de la Grèce ( $30 \mathrm{H}$ en tout), et en plus une ou même deux langues vivantes $(120-240 \mathrm{H})$, et d'autres matières qui sont optionnelles ou facultatives, selon le choix de 1 'étudiant (épigraphie, mythologie, philosophie, histoire de l'art ancien).

Les Universités de Prague et de Brno offrent également l'enseignement du grec comme matière principale, avec un peu de latin $(120-240 \mathrm{H})$. Dans ce cas le latin et le grec peuvent être associés. On peut donc recevoir le diplôme des "Etudes latines et Etudes grecques " ou bien " Etudes latines et Histoire » ou bien "Etudes latines et Etudes française », ou bien «Etudes latines et Etudes Tchèques » ou encore «Etudes grecques et Philosophie ", etc. Aucune des deux matières n'est dominante sauf le fait que les étudiants rédigent un 
travail écrit seulement dans une matière principale (selon leur choix). Ce travail est un mémoire de 70 à 100 pages et il est soutenu devant le jury.

Les Universités de Brno et de Bratislava offrent également un diplôme de «Philologie classique » qui combine le latin et le grec, sans autres matières principales.

Chaque année, quelques étudiants bénéficient de la possibilité de faire une année ou plus à l'étranger (Erasmus, co-tutelle). Pendant l'année universitaire 2004/2005, deux étudiants pragois en grec, ont fait leurs études, grâce à Erasmus, dans les pays francophones, l'une à Toulouse, l'autre à Liège; une ancienne étudiante en latin a terminé ses études doctorales en co-tutelle à la Sorbonne chez Madame le Professeur M. Fruyt et un autre étudiant en latin va terminer sa co-tutelle chez Monsieur le Professeur J.-L. Fredouille.

\section{Les cours de langues et littératures anciennes pour étudiants d'autres spécialités universitaires}

Comme peu d'étudiants ont fait du latin au lycée, ils doivent rattraper à l'Université. Il s 'ensuit que l'enseignement du latin (rarement du grec) fait partie, souvent de façon obligatoire, de plusieurs autres cursus, même si ce n'est que pour 30 à $60 \mathrm{H}$ en tout :

- latin en Faculté des Lettres dans des cursus d'histoire, études d'archiviste, archéologie classique (latin et grec), sciences religieuses, philosophie (optionnel entre le grec et le latin), études françaises, italiennes et espagnoles ; à Prague aussi dans les cursus d'histoire de l'art, études allemandes, musicologie, études tchèques.

- dans les autres facultés : en Facultés de Médecine, de Sciences humaines, de Pharmacie, de Droit, de Médecine Vétérinaire, de Théologie (latin et grec), en Faculté pédagogique.

En général, ces étudiants fréquentent des cours spéciaux, assurés, selon les Universités, soit par le département d'Etudes classiques de la Faculté des Lettres, soit par le Centre des langues étrangères de la Faculté en question, soit par des personnes du département en question (philosophie), mais ils peuvent assister aussi aux mêmes cours que les étudiants spécialistes; ceci est pourtant assez rare car ils n'ont pas le niveau de connaissances langagières nécessaire. On organise aussi des cours d'histoire ancienne, de littérature, mythologie, civilisation etc. (cela dépend des Universités) pour les étudiants non spécialistes.

\section{En Pologne}

23 Ce pays de près de quarante millions d'habitants ne compte pas moins d'une dizaine d'universités comportant un cursus de langues anciennes de cinq ans et des études doctorales. L'enseignement du latin y semble particulièrement développé.

\section{L'enseignement secondaire}

Le grec ancien serait seulement étudié en lycée à Krakow, Warsaw et Gdansk ; le latin l'est dans un certain nombre de lycées, à raison d'environ deux heures hebdomadaires pendant deux ou trois ans, mais les lycéens latinistes ne représentent que 3\% de leur classe d'âge. Et le nombre de lycées où le latin est enseigné tend à diminuer.

Certaines universités ont encore un examen d'entrée, mais celui-ci sera supprimé partout à la rentrée prochaine avec l'instauration d'un examen de fin d'études secondaires. 


\section{La formation au métier d'enseignant et le recrutement des professeurs}

Un professeur qui a un diplôme de philologie classique n'enseigne que le latin, sauf s'il a aussi des diplômes dans d'autres matières. Il n'existe pas de Faculté pédagogique pour assurer la formation professionnelle initiale ni assurer l'insertion professionnelle des diplômés. Il ne semble pas qu'il y ait non plus de concours de recrutement. Neuf étudiants sur dix ont suivi, en plus de leurs études classiques, un enseignement optionnel de pédagogie et didactique en troisième et quatrième années, nécessaire pour pouvoir accéder à l'enseignement au lycée.

\section{Le cursus universitaire de spécialité Etudes Classiques}

27 A l'université, la filière d'études classiques associe nécessairement latin et grec dans la même formation. Celle-ci dure actuellement cinq ans, mais le pays va passer bientôt au système L/M/D. Les matières enseignées dans le diplôme de philologie classique concernent seulement les Sciences de l'Antiquité.

A leur entrée en filière de philologie classique, les étudiants sont tous débutants en grec, et la moitié d'entre eux l'est en latin. Pour remédier à la différence de niveau avec ceux qui ont déjà fait 150 à 200 heures de latin au lycée, l'enseignement est plus intensif pour les latinistes débutants. L'université de Poznan a mis au point pour eux un système original d'apprentissage intensif du latin (20H par semaine la première année) en reportant pour eux le début de l'étude du grec en deuxième année et en allégeant quelque peu celle-ci (voir tableau des horaires ci-dessous). Au bout d'un an d'études, les étudiants doivent être capables de lire des textes de César et Cicéron ou, en grec, Xénophon ou Platon.

Horaires à l'U. de Poznan

\begin{tabular}{|l|l|l|l|l|}
\hline POZNAN & & $1^{\circ}+2^{\circ}+3^{\circ}$ années & $4^{\circ}+5^{\circ}$ années & Total \\
\hline débutants & $\mathrm{L}$ & $432+240+150$ & $150+100$ & 1072 \\
\hline \hline en latin & $\mathrm{G}$ & $0+240+180$ & $130+60$ & 610 \\
\hline TOTAL & $\mathrm{L}+\mathrm{G}$ & $432+480+330$ & $280+160$ & \\
\hline \hline Avancés & $\mathrm{L}$ & $240+210+180$ & $180+100$ & 910 \\
\hline en latin & $\mathrm{G}$ & $240+210+150$ & $150+60$ & 810 \\
\hline TOTAL & $\mathrm{L}+\mathrm{G}$ & $480+420+330$ & $330+160$ & \\
\hline
\end{tabular}

Au bout des cinq années, quel que soit son niveau de départ et son université, tout étudiant semble avoir pu suivre 1600 à 1700 heures d'enseignement en langue et littérature latines et grecques et disciplines auxiliaires des études classiques. La proportion entre cours de grec et cours de latin semble à peu près égale à l'U. de Krakow, mais varie de 3 à 4 aux U. de Torun et Poznan et à l'U. Catholique de Lublin, en faveur du latin. 

nombre est plus grand à Poznan. A Gdansk, en revanche, si la possibilité existe, cela ne s'est pas encore produit. Le principal pays d'accueil est l'Allemagne, suivi par l'Italie et la Grèce.

\section{Les cours de langues et littératures anciennes pour étudiants d'autres spécialités universitaires}

31 Certaines formulations de nos correspondants donnent à penser qu'une petite partie de leur public de «spécialistes " peut être aussi constituée d'étudiants ayant choisi le latin comme discipline «mineure » dans d'autres spécialisations (probablement sous la forme d'une option suivie sur plusieurs semestres).

Pour les non-spécialistes, des cours de littérature sur textes traduits sont donnés dans tous les départements de philologies modernes, mais avec des volumes horaires assez faibles (une quarantaine d'heures au total à Torun), et aussi en histoire à Gdansk.

Ce qui est en revanche impressionnant dans les universités polonaises, c'est la vitalité de l'enseignement de la langue latine dans les autres filières d'études. Par exemple, les instituts de philologie classique de l'université de Gdansk et de l'université catholique de Lublin dispensent des cours de langue latine pour non-spécialistes dans à peu près quinze autres départements : dans les cursus de philologie polonaise, de philologie allemande, de philologie anglaise, de philologie slave, d'histoire, d'archéologie, et bien sûr, pour l'université catholique de Lublin, à l'Institut d'histoire de l'Eglise (Faculté de théologie). Un cours de grec s'y ajoute en philologie slave. Pour la Faculté de philosophie, il est offert un cours de langue grecque et un cours de langue latine. Il y a aussi à l'université de Lublin un enseignement de latin d'un semestre pour les étudiants de l'Institut des Arts, de l'Institut pédagogique de la Faculté des sciences sociales, de l'Institut d'administration et marketing, de l'Institut d'études politiques et juridiques de la Faculté de droit, et aussi à la Faculté de droit international et d'économie. Enfin, pour la Faculté d'études pratiques de langues étrangères, il est proposé un cours de grec débutant et un nombre impressionnant de cours de latin débutant, sur plusieurs années, totalisant près de huit cents heures. L'inventaire est à peu près le même aux universités de Krakow, Poznan et Torun.La principale différence (mais c'est un enjeu considérable) est le caractère obligatoire ou facultatif de cet enseignement: le latin est obligatoire à peu près dans toutes les filières à Torun et Gdansk, et probablement aussi à Lublin (vu les volumes horaires), facultatif ailleurs (sauf en histoire et archéologie, où la nécessité de connaître le latin et l'utilité de connaître le grec ne semblent nulle part remises en cause). Dans les villes où il existe des facultés de médecine et de pharmacie, des cours de latin sont proposés aussi dans ces cursus, voire même imposés (par ex. à Gdansk). Là où l'enseignement des langues anciennes est optionnel, et s'il s'adresse à des étudiants littéraires ou philosophes, ces étudiants suivent les mêmes cours que les étudiants d'études classiques (Poznan).

L'enseignement de la langue latine dans une «faculté d'études pratiques de langues étrangères » (Lublin) ou un "collège des langues étrangères » (Poznan), c'est-à-dire comme option à l'égal des langues vivantes, est aussi un trait original. 


\section{En Slovénie}

Il y a une seule université avec un Département de Philologie Classique, celle de Ljubljana, fondée en 1919 en même temps que l'Université.

\section{L’enseignement secondaire}

Les étudiants désireux d'avoir des connaissances du grec avant l'Université ont la possibilité d'en faire dans deux lycées à Ljubljana pendant quatre ans à raison de 2,5 $\mathrm{H}$ par semaine. Quant au latin, l'enseignement est offert dans cinq écoles élémentaires (de 6 à 15 ans) - en tout 118 élèves - et six lycées (de 15 à 18 ans) publics et quatre lycées catholiques privés.

\section{La formation au métier d'enseignement et le recrutement des professeurs}

37 Pour devenir professeurs du lycée, les étudiants ne fréquentent pas les facultés pédagogiques ; les professeurs enseignent deux matières, l'association du grec et du latin étant rare.

\section{Le cursus universitaires de spécialité Latin ou Grec} troisième cycle de trois ans. L'enseignement de la langue maternelle n'est pas compris dans le cursus; par contre, y entrent histoire ancienne, philosophie ancienne, mythologie, patristique.

41 Les études font $240 \mathrm{H}$ dans le cursus de grec et $240 \mathrm{H}$ dans le cursus de latin (langue + linguistique + littérature) par an; en quatre ans d'études, pour devenir professeur de lycée, les étudiants ont donc suivi environ $1200 \mathrm{H}$ en grec ou $1200 \mathrm{H}$ en latin.

\section{Les cours de langues et littératures anciennes pour les étudiants d'autres} spécialités universitaires

L'apprentissage du latin est compris dans les cursus d'histoire, archéologie classique, études françaises, italiennes et espagnoles, d'histoire de l'art, de médecine et de théologie; en linguistique comparée, on étudie le latin ainsi que le grec. En général, ces étudiants fréquentent des cours spéciaux, donc pas les mêmes cours que les spécialistes. spécialistes (cursus de littérature comparée). 


\section{En Estonie}

Une seule Université a un Département de Lettres Classiques, celle de Tartu. Cette université, fondée en 1632, a eu une histoire très mouvementée. Les études classiques, introduites au XIXe siècle, ont été supprimées en 1954 par le régime soviétique, pour être rouvertes en 1990.

\section{L'enseignement secondaire}

Il n'y a aucune possibilité d'étudier la langue et littérature grecques avant l'université ; la langue latine est enseignée dans certains lycées, $d$ 'habitude pendant deux ans.

\section{La formation au métier d'enseignant et le recrutement des professeurs}

47 Pour devenir professeur du lycée, il faut suivre pendant une année une formation en Faculté pédagogique.

\section{Le cursus universitaire de spécialité Latin ou Grec}

Aucune connaissance préalable de grec ni de latin n'est requise. Il existe pourtant un examen d'entrée qui se compose d'un test et d'un interview.

Organisation et durée des études : $1^{\circ}$ cycle $: 3$ ans (auparavant 4 ans), $2^{\circ}$ cycle $: 2$ ans, $3^{\circ}$ cycle : 4 ans.

En licence, le grec et le latin sont joints dans le même cursus que l'on appelle « Philologie classique »; en cursus de magister et de doctorat, ils sont étudiés séparément. Cependant, les étudiants ont toujours la possibilité d'étudier encore une matière principale en plus, ce qui leur offre une meilleure chance de trouver un débouché satisfaisant. A côté des cours sur les langues et littératures latines et grecques, d'autres disciplines sont proposées, soit obligatoires (histoire ancienne, philosophie ancienne, mythologie, cours de langue anglaise et allemande), soit optionnelles (antiquité tardive).

51 Le nombre d'heures d'enseignement est d'environ $700 \mathrm{H}$ par an en grec et latin. Les études à l'étranger sont possibles et réalisées.

\section{Les cours de langues et littératures anciennes pour les étudiants d'autres spécialités universitaires}

52 Le latin est obligatoire pour les étudiants des cursus d'Etudes françaises, d'Etudes italiennes, d'Etudes espagnoles, et pour les étudiants des Facultés de Médecine et de Droit; il est optionnel pour les cursus de Philosophie et d'Histoire. Le grec néotestamentaire est obligatoire pour les étudiants de Théologie.

53 En général, ces étudiants ne fréquentent pas les mêmes cours que les spécialistes. On organise des cours pour les étudiants des autres départements (où la connaissance des langues classiques n'est pas requise), surtout en mythologie et en architecture anciennes. 


\section{En Lettonie} Une seule Univers
fondée en 1919.

\section{L’enseignement secondaire}

Les étudiants du secondaire acquièrent certaines connaissances de littératures grecque et latine (deux à trois mois dans certains lycées); la langue grecque n'est pas du tout enseignée dans le secondaire, la langue latine l'est dans quelques lycées (deux à trois ans, en option).

\section{La formation au métier d'enseignant et le recrutement des professeurs}

Pour devenir professeur du lycée, il faut faire une année de Faculté pédagogique.

\section{Le cursus universitaire de spécialité Latin ou Grec}

57

Il n'y pas d'examen d'entrée et aucune connaissance préalable de grec ni de latin n'est requise. Il n'y a qu'un seul diplôme, celui de Philologie classique; le grec et le latin ne peuvent ni être étudiés séparément ni en association avec une autre matière principale. $\mathrm{L}$ 'organisation des études correspond au système L/M/D $\left(1^{\circ}\right.$ cycle $=3$ années, $2^{\circ}$ cycle $=2$ années, $3^{\circ}$ cycle $=3$ années).

58 A côté des cours sur les langues et littératures latines et grecques, d'autres disciplines sont proposées: histoire ancienne, mythologie (obligatoire), philosophie ancienne, réception de l'héritage classique (option).

Le nombre d'heures pour une matière est d'environ $300 \mathrm{H}$ dans le cursus du grec et $300 \mathrm{H}$ dans le cursus du latin par an, soit environ $1800 \mathrm{H}$ en premier cycle de Philologie classique. Les études à l'étranger sont possibles et réalisées.

\section{Les cours de langues et littératures anciennes pour les étudiants d'autres spécialités universitaires}

60 Le latin est proposé aux étudiants en histoire, en archéologie classique, en langue française (les filières études italiennes et études espagnoles n'existent pas à l'Université de Riga) et en faculté de médecine (un semestre). Le latin et le grec sont obligatoires en théologie et optionnels en philosophie.

61 En général, ces étudiants fréquentent des cours qui ne sont pas ceux des étudiants spécialistes. On organise aussi, pour les étudiants des autres départements, des cours où la connaissance des langues classiques n'est pas requise.

\section{En Hongrie}

Il existe des départements de lettres classiques dans cinq universités avec une formation de cinq ans : Université ELTE à Budapest, Université de Debrecen, Université de Szeged, Université de Pécs, Université Catholique Pierre Pazmany de Piliscsaba. Il existe aussi un 
département de lettres classiques à l'université de Miskolc, mais il ne délivre pas de diplôme de professeur de latin.

\section{L'enseignement secondaire} semaines, 9 à $12 \mathrm{H}$ par semaine), et $600 \mathrm{H}$ pour les deux années du cycle de spécialité (soit 10 à $12 \mathrm{H}$ par semaine). Soit environ $1300 \mathrm{H}$ pour chacune des deux filières.

71 première année est exigeant (cours de grammaire et lectures de textes tels que le Criton 
de Platon). Certaines universités, comme l'université catholique Pierre Pazmany, prévoient que l'étudiant dépourvu de toute connaissance préalable en grec doit obtenir d'abord tout ou partie des trois cours d'initiation à la langue grecque du cursus des latinistes avant de commencer le cursus.

Les diplômés de cinquième année ont la possibilité de s'inscrire à une école de doctorat, où la formation de base dure trois ans, et qui s'achève par les examens de doctorat oraux. Puis, l'étudiant a un délai de deux ans pour élaborer la thèse et la soutenir pour obtenir la dénomination PhD. En Hongrie, il existe trois écoles de doctorat en lettres classiques (Université ELTE de Budapest, Université de Debrecen, Universités de Szeged et de Piliscsaba associées).

\section{Les cours de langue et littératures anciennes pour les étudiants d'autres spécialités} universitaires

73 Étant donné que le hongrois appartient aux langues finno-ougriennes, pour les Hongrois le savoir ou au moins les connaissances de base en latin sont une ouverture très importante pour beaucoup de disciplines dont le vocabulaire est d'origine latine. Ainsi le latin est enseigné dans plusieurs facultés, de façon obligatoire ou optionnelle.

Depuis les années cinquante le nombre des élèves apprenant le latin au lycée a été bien réduit. A cause d'un manque de connaissance minimale en langue latine, une bonne partie des chercheurs a été exclue de l'étude des sources historiques et, d'autre part, les étudiants ont eu des problèmes au début de leurs études, surtout en sciences naturelles. Ainsi il est devenu nécessaire d'enseigner le latin dans le cadre de l'enseignement supérieur, dans certaines facultés et écoles supérieures. Dans ces parcours d'étude, il faut apprendre un minimum de vocabulaire, les bases de la grammaire latine, surtout dans le domaine de la morphologie des noms, les règles de la dérivation et il faut comprendre des phrases latines très simples.

75 Les institutions concernées peuvent être classées en deux groupes selon que l'apprentissage du latin y est obligatoire ou non. Des connaissances assez approfondies sont exigées en faculté de droit $(60 \mathrm{H})$, en histoire et archéologie $(90 \mathrm{H})$, en langues néolatines $(180 \mathrm{H})$, en faculté de théologie, dans les séminaires catholiques ou protestants. La formation est moins approfondie ( 30 à $60 \mathrm{H}$ ) dans les instituts de formation musicale de spécialistes de musique sacrée, les facultés de médecine, de médecine dentaire, pharmacologie, certaines écoles supérieures sanitaires. L'apprentissage du latin est facultatif en biologie, lettres et langues vivantes.

\section{À Chypre}

Pour comprendre la situation à Chypre, il faut garder à l'esprit cette évidence que le grec de Platon n'est autre que la forme ancienne de leur langue maternelle, et que son étude dans ce pays tient plus de place que celle du latin en France, et y est mieux considérée.

\section{L'enseignement secondaire}

77 La langue et la littérature grecques de l'Antiquité peuvent être étudiées pendant six ans, alors que l'étude du latin n'est offerte que pendant les trois années de lycée. On peut aussi ne commencer le grec ancien qu'au lycée. 


\section{La préparation au métier d'enseignant et le recrutement des professeurs}

78 Après leurs études classiques, les étudiants suivent les cours d'une Faculté pédagogique pendant un an. N'y entrent que ceux qui sont reçus à un concours national.

\section{Les cursus universitaires à spécialité Etudes Classiques}

79 Il y a à l'Université de Nicosie, qui est la seule de Chypre, quatre filières qui comportent un enseignement obligatoire plus ou moins important des langues et littératures grecques et latines: Etudes classiques (associant grec ancien et latin), Philosophie, Histoire et archéologie, Etudes byzantines.

On ne peut entrer en premier cycle et en second cycle qu'après un examen. Le premier cycle dure quatre ans, le second deux ans, le troisième (doctorat) cinq ans. Une réforme est prévue dans un proche avenir.

81 En «Etudes classiques" au sens strict, il y a une trentaine d'étudiants par année en premier cycle, une quinzaine par année en second cycle, et quatre en doctorat (au total environ 150).

Chaque "cours» représente trois heures hebdomadaires pendant un semestre. Les enseignements du premier cycle d'études classiques totalisent quinze à vingt heures hebdomadaires.

83 Pendant les huit semestres du premier cycle d'études classiques, il est donné treize cours de langue et littérature grecques pour la période antique, un pour l'époque byzantine, six pour l'époque moderne. Et huit cours de langue et littérature latines. A quoi s'ajoutent quatre cours de linguistique et quatre cours d'histoire, deux cours de philosophie, trois cours de langue étrangère et quatre cours d'option. Ce sont les enseignements de littérature et de linguistique qui dominent. Durant ce premier cycle de quatre ans, les étudiants suivent donc environ 500 heures de grec ancien et 300 heures de latin, sur un total d'environ 1700 heures.

Quelle que soit leur filière, tous les étudiants des quatre filières où les langues et littératures grecques ou latines ont une large part suivent les mêmes cours que les étudiants de la filière "Etudes classiques». Leur «mineure» (ou en grec, ou en latin) comporte au moins dix cours de trois heures hebdomadaires répartis sur les huit premiers semestres (trois de langue, cinq de littérature, deux d'histoire), soit un peu plus de 350 heures. Les possibilités d'échange«Erasmus / Socrates» sont assez souvent utilisées pour un ou deux semestres.

Les cours de langues et littératures anciennes pour étudiants d'autres spécialités universitaires

L'enseignement du grec ancien ou du latin est proposé comme option dans quelques filières comme les Etudes françaises. Les langues anciennes ne sont pas enseignées en Faculté des sciences. 


\section{Essai de bilan}

\section{Diversité des contenus d'enseignement en sciences de l'Antiquité}

Selon les pays, l'enseignement d'une langue ancienne inclut des contenus différents. Il y a bien sûr toujours principalement, en grec comme en latin, une suite de cours de pratique de la langue et de lecture et analyse des textes originaux (15 à 20 œuvres ou groupements de textes étudiés en cinq ans dans chaque langue), à quoi s'ajoutent une série de cours d'histoire de la littérature (auxquels l'assiduité n'est pas toujours obligatoire).

Les textes les plus étudiés appartiennent aux époques classique, hellénistique et impériale. Les textes de patristique et les textes d'époque médiévale ou humaniste font l'objet d'options ou de cours de master; ces enseignements ne sont pas assurés dans toutes les universités, et là où ils le sont, ils ne sont pas toujours ouverts chaque année.

Il s'y ajoute souvent, en fin de cursus, des cours (obligatoires ou optionnels, selon les universités) de philologie et d'histoire de la philologie classique. Il peut s'y ajouter aussi, à chaque fois à raison d'un semestre, des cours de rhétorique antique, d'esthétique antique, d'histoire ancienne (le plus souvent obligatoires), d'histoire de l'art, d'archéologie, de philosophie, ou encore d'histoire religieuse.

89 L'étude de la littérature et de la langue est partout complétée par des cours spécifiques de grammaire normative, d'histoire de la langue, de grammaire comparée, de stylistique, de métrique, et parfois de linguistique. Au vu des volumes horaires et de l'inventaire des cours, il semble qu'en Pologne et en Hongrie, et peut-être aussi dans d'autres pays où les deux matières sont couplées, l'étude du grec et celle du latin n'obéissent pas aux mêmes objectifs. Il semble que l'étude du latin vise une compréhension fine de la langue du point de vue grammatical et stylistique (il y a au moins un cours par semestre dans l'un de ces deux domaines en latin, et souvent dans chacun des deux) alors que celle du grec semble davantage axée sur la littérature, l'histoire et l'histoire des idées. Ce qui se comprend si l'on se souvient que le latin est la seule langue dont la plupart des étudiants feront un usage professionnel en devenant enseignants du second degré, alors que le grec apporte plutôt des fondements culturels.

\section{Les débouchés des étudiants latinistes ou hellénistes}

90 Un débouché important est partout l'enseignement du latin dans les lycées. Mais ce n'est pas toujours celui qui canalise les plus gros effectifs (en Lettonie et Estonie, seulement un ou deux étudiants par an). Il faut aussi un bon nombre de professeurs pour l'enseignement du latin aux étudiants non-spécialistes dans les universités, là cet enseignement est obligatoire et où, par conséquent, les effectifs sont importants; la plupart des professeurs qui assurent ces cours ne semblent pas être des enseignantschercheurs des départements d'études classiques (sauf en heures supplémentaires), ni des enseignants sélectionnés par eux.

D'autre part, dans les pays où le cursus universitaire dure cinq ans, un diplômé littéraire, quelles que soient les matières qu'il a étudiées, est considéré comme une personne de haut niveau d'instruction, apte à tout métier où une vaste culture, d'excellentes compétences langagières et de bonnes capacités de raisonnement et d'organisation sont souhaitées. Bien sûr, surtout si les études ont associé seulement latin et grec, les 
principaux débouchés restent culturels: métiers de la documentation et des bibliothèques, musées, travail archéologique, traducteurs, journalisme, personnels des maisons d'édition (rédacteurs, correcteurs), même si on signale aussi certains postes de haute administration. Le niveau Doctorat ou Ph.D. ouvre sur les recrutements dans l'enseignement supérieur et des équipes de recherche. Par exemple, en Pologne, l'Académie des Arts (recherche archéologique), l'Académie des Sciences (histoire des sciences), l'Atelier du Lexicon du latin médiéval. Dans certains pays, comme la Hongrie, la Pologne, la Tchéquie, la recherche est vivace non seulement dans le domaine de l'Antiquité mais aussi sur les textes et documents en latin des époques médiévale et humaniste, ce qui élargit le domaine de recrutement des docteurs latinistes.

Là où la formation est consacrée exclusivement au latin et au grec ancien, nos correspondants (par exemple les polonais) craignent que les débouchés dans l'enseignement semblent devoir se raréfier. Là où le latin (ou le grec) n'est qu'une des deux matières étudiées, l'autre étant plus «moderne» (i.e. en Hongrie, Tchéquie, Slovaquie, Slovénie), les perspectives sont évidemment plus ouvertes, tant dans l'enseignement que dans les métiers de culture ou d'administration.

Chypre constitue un cas à part. La langue qui ouvre des débouchés est cette fois le grec ancien, et ces débouchés sont relativement importants en nombre et en diversité : il s'agit toujours principalement du professorat, des métiers des archives et des bibliothèques, de l'archéologie, du journalisme, et de tous les métiers du livre. Mais dans tous ces domaines, avoir étudié la langue de l'Antiquité est, d'après notre informatrice, un facteur de valorisation du dossier professionnel, qui permet de trouver plus facilement du travail et d'être mieux rémunéré. D'après notre informatrice, l'Université de Nicosie fournit chaque année trente à quarante diplômés spécialistes de grec ancien et de latin (de premier cycle et de second cycle) et cela correspond aux besoins du marché de l'emploi.

\section{Quelques corrélations significatives}

Si nous avons bien interprété les informations de nos correspondants, là où il y a abondance de cours de langue latine pour non-spécialistes à l'Université, c'est que très peu de jeunes ont étudié le latin au lycée et qu'eux-mêmes ou leurs professeurs sentent la nécessité d'une connaissance minimale du lexique (pour les études scientifiques ou juridiques) ou des structures fondamentales de la langue (pour les départements d'études littéraires et de langues vivantes). Cette floraison de cours n'est donc que la compensation, minimale et ultime, d'une situation de faiblesse de l'enseignement des langues anciennes dans l'enseignement secondaire.

Pour la formation des spécialistes en études latines et/ou en études grecques, ces différents pays présentent trois façons très différentes d'organiser les contenus du cursus universitaire, qui ne sont pas sans incidence sur les débouchés (et en retour sur l'attractivité de ces enseignements et leur fréquentation) :

96 - le cursus polonais dure cinq ans; il ne comporte que des Sciences de l'Antiquité mais associe grec et latin ; ce pays ne connait pas de recrutement par concours des enseignants $\mathrm{du}$ secondaire, mais exige des futurs enseignants le suivi d'un minimum de formation pédagogique en plus du diplôme disciplinaire ;

97 - d'autres pays (Hongrie, Slovaquie, Slovénie, Tchéquie [Prague]) ont aussi un cursus de cinq années indissociables (toutefois les universités tchèques autres que celle de Prague 
offrent un diplôme aussi à la fin des trois premières années, et tous ces pays vont passer au système L/M/D d'ici quelques années) mais on y pratique une bi-valence disciplinaire qui fait que les cursus de latin et de grec sont très rarement suivis conjointement par le même étudiant, mais qu'il étudie une autre discipline dont les débouchés professionnels sont plus larges (même dans le domaine de l'enseignement); ces pays ne connaissent pas non plus le recrutement par concours des enseignants du secondaire, et exigent des futurs enseignants le suivi d'un minimum de formation pédagogique en plus du diplôme bi-disciplinaire ;

98 - plus proches de la situation française actuelle, d'autres pays (Chypre, pays baltes) distinguent deux cycles et associent à l'enseignement des Sciences de l'Antiquité d'autres matières qui sont utiles quelle que soit la formation (comme les langues vivantes ou la langue maternelle) et des options diversifiées ou une "mineure" dans une autre discipline ; les futurs professeurs fréquentent ensuite une faculté pédagogique et l'avenir professionnel est assuré pour les lauréats du concours (d'entrée ou de sortie) de ces Facultés.

\section{AUTEURS}

\section{MICHĖLE BIRAUD}

Université de Nice

DAGMAR MUCHNOVA

Université de Prague 